\title{
La prensa costarricense ante el conflicto: el caso del proceso de concesión de los puertos de JAPDEVA (2010-2011)
}

\author{
The conflict over the privatization of JAPDEVA's ports in the Costa Rican press
}

(2010-2011)

\section{José Andrés Díaz González}

Universidad Nacional y Universidad de Costa Rica, Costa Rica jose.diaz.gonzalez@una.cr joseandres.diaz@ucr.ac.cr

doi: http://dx.doi.org/10.15359/abra.35-50.4

\begin{abstract}
Resumen: Se analiza el contenido de las noticias publicadas por cuatro medios de prensa escrita: el Semanario Universidad, El País, Diario Extra y La Nación, en relación con el proceso de privatización de los puertos administrados por la Junta de Administración Portuaria y Desarrollo Económica de la Vertiente Atlántica (JAPDEVA). Se concluye que existen dos narrativas dominantes en el contenido de los medios: la primera, a favor del proceso de privatización; la segunda defiende un proceso de modernización portuaria sin su privatización. En ambas narrativas se oculta o excluye los problemas de fondo que han originado el conflicto y no toman en cuenta las acciones y opiniones de las personas trabajadoras de los puertos ni a la población costarricense sobre este tema.
\end{abstract}

Palabras claves: Medios, Sindicatos, Prensa, Análisis de Contenido, Puertos, Privatización, Modernización

\begin{abstract}
This paper analyzes the content of the news published by four newspapers: Semanario Universidad, El País, Diario Extra and La Nación, regarding the privatization process of the ports managed by the Board of Port Administration and Economic Development of the Atlantic (JAPDEVA-"Junta de Administración Portuaria y Desarrollo Económica de la Vertiente Atlántica"). Two main discourses are found in the news content: the first discourse supports the privatization process, while the second one advocates for port modernization without privatization. Both discourses exclude or conceal the underlying problems that originated the conflict and do not take into consideration the actions and opinions of port workers and the Costa Rican population regarding this topic.
\end{abstract}

Keywords: Mass Media, Unions, Press, Content Analysis, Ports, Privatization, Modernization

\footnotetext{
${ }^{1}$ El presente artículo presenta algunas conclusiones de un proyecto de investigación mayor, titulado: "Análisis político del discurso informativo de la prensa escrita sobre los movimientos sociales en Costa Rica (Fase II)", realizado en manera conjunta por el Programa Umbral Político del Instituto de Estudios Sociales en Población (IDESPO) de la Universidad Nacional de Costa Rica (UNA) y la Escuela de Ciencias Políticas de la Universidad de Costa Rica (UCR) durante el 2012.

${ }^{2}$ Licenciado en Ciencia Política y Mágister en Historia, ambos por la Universidad de Costa Rica (UCR). Profesor e Investigador de la Escuela de Ciencias Políticas (UCR) e investigador en el Instituto de Estudios Sociales en Población (IDESPO) de la Universidad Nacional (UNA).
} 
Fecha de recepción: 26-08-2014 Fechas de reenvíos: 16-04-15/22-04-2015/01-06-2015/01-06-2015/02-06-2015 Fecha de aceptación: 14-04-2015 Fecha de publicación: 30-06-2015.

En el presente artículo se realiza un análisis de contenido de las noticias publicadas por el Semanario Universidad (SU), El País (EP), Diario Extra (DE) y La Nación (LN) sobre el proceso de privatización de los puertos administrados por la Junta de Administración Portuaria y Desarrollo Económica de la Vertiente Atlántica (JAPDEVA) y el conflicto surgido en torno a este, durante el primer año de la Administración de Laura Chinchilla Miranda (8 de mayo del 2010 al 7 de mayo del 2011). De esta manera el artículo realiza un análisis de contenido con el propósito de identificar y analizar cuál fue el énfasis y la forma en que cada medio desarrolla la información publicada en relación con el proceso de modernización de JAPDEVA, qué actores identifican y cuál es la visión general del conflicto que buscan mostrar a la opinión pública.

La importancia en determinar la imagen proyectada por los medios se debe a que se parte de la idea de que ellos presentan o construyen el contenido de sus noticias para que estas respondan a su propia lógica de interpretación de los acontecimientos que reseñan:

El contenido de los medios se puede basar en lo que sucede en el mundo material aunque separa y sólo destaca ciertos elementos, y la propia estructura lógica del medio se impone sobre dichos elementos. La realidad es manipulada necesariamente cuando los sucesos y las personas se vuelven a colocar dentro de noticias transmitidas... Los medios pueden imponer su propia lógica al ensamblar materiales en una serie de maneras, incluyéndose el énfasis de ciertos comportamientos y personas y estereotiparlas... Una de las maneras más obvias en que el contenido de los medios estructura un ambiente simbólico es poniéndole una mayor atención (en la forma de más tiempo, mayor importancia y demás) a ciertos sucesos, personas, grupos y lugares que a otros (Shoemaker y Reese, 1994, p. 38).

Asimismo, no hay que olvidar además que las noticias, como una unidad de información que transmite el medio de comunicación no solamente se encuentra definida por el ente emisor, sino también por el receptor (opinión pública) que tiene interés o encuentra cierto valor de informarse sobre una serie de acontecimientos determinados (McQuail, 1996, p. 372). Por lo tanto, el seguimiento que realizan los periódicos sobre el conflicto generado en torno al proceso de concesión de los puertos de JAPDEVA, puede también interpretarse como la existencia en un interés de la población costarricense sobre este.

Además, no hay que obviar el hecho de que si bien los medios de comunicación no le indican a la población cómo pensar, sí pueden influir indicándoles en qué pensar (Bustos Mora, 2011, p. 111), en otras palabras, los medios pueden desarrollar una agenda propia con el propósito de colocar la atención de la opinión pública sobre determinados temas, al mismo tiempo que se ignoran otros. 


\section{Breve cronología del conflicto sobre JAPDEVA}

El conflicto sobre JAPDEVA tiene su origen en abril del 2006, cuando Oscar Arias Sánchez, quién asumía la Presidencia de la República en mayo de ese año; anuncia a un grupo de empresarios extranjeros su intención de concesionar los puertos estatales (Araya, 2011a). Como respuesta a estas declaraciones, en agosto del 2006, el Sindicato de Trabajadores de la Junta de Administración Portuaria y Desarrollo Económica de la Vertiente Atlántica (SINTRAJAP) envía a la administración Arias Sánchez una propuesta de modernización de los puertos, que no implicaba su privatización, la cual es rechazada.

En el 2007 se acrecientan los conflictos entre SINTRAJAP y la administración de JAPDEVA debido al incumplimiento de pagos de horas extra y otros compromisos adquiridos con los trabajadores y trabajadoras de la institución. Asimismo, el sindicato denuncia ese año que desde algunos medios de comunicación se lleva a cabo una campaña en su contra con el propósito de poner a la opinión pública en favor del proceso de concesión y en contra de las solicitudes del sindicato ${ }^{3}$.

En febrero del 2008 la administración Arias Sánchez inicia formalmente el proceso de concesión de los puertos y, dada la reticencia de la mayoría de trabajadores y trabajadoras a este proceso, el gobierno informa que estaría dispuesto a darles hasta USD80 millones de dólares como indemnización (Espinoza, 2008), lo cual SINTRAJAP interpreta como un soborno o "torcedura de brazos" con el propósito de que permitan entregar los puertos a manos privadas (Informa-tico.com, 2008).

En junio del 2009 un grupo de trabajadores de JAPDEVA forman el denominado "Grupo Mediador", al cual SINTRAJAP acusa de responder a los intereses de la administración Arias Sánchez. El Grupo Mediador se da a la tarea de recoger firmas de las personas miembros del sindicato con el propósito de convocar una Asamblea Extraordinaria para conocer la propuesta de indemnización realizada por el gobierno, al mismo tiempo que la misma sea aceptada. Sin embargo, la iniciativa de este grupo no prosperó (Rojas, 2009 y Villalobos, 2009).

El 15 de enero del 2010 el Grupo Mediador convoca a una reunión de trabajadores, la cual denomina "Asamblea General de SINTRAJAP" y en la que se acuerda destituir a la junta directiva de SINTRAJAP que hasta ese momento era presidida por Rolando Blear y, en lugar, nombrar a Douglas Brenes como presidente del sindicato. El Ministerio de Trabajo avala dicha reunión e inscribe a la nueva junta directiva.

Lo anterior lleva a la junta depuesta, a la Asociación Nacional de Empleados Públicos y Privados (ANEP) y al Partido Frente Amplio (FA) a presentar distintos recursos de amparo ante la Sala Constitucional, los cuales son declarados con lugar el 25 de agosto del 2010; lo que obligó la restitución de la junta directiva presidida por Blear y anuló todos los actos llevados a cabo por la junta directiva presidida por Brenes, incluso una nueva

${ }^{3}$ Al respecto, SINTRAJAP construye una cronología de los hechos más importantes para ellos en relación con este conflicto, la cual puede consultarse en: http://www.sintrajap.com/?p=2969 
Convención Colectiva que había sido firmada entre estos y la administración de JAPDEVA y que facilitaba el proceso de concesión de los puertos (Sala Constitucional, voto $14192-10$ y voto $14193-10)$.

En enero del 2011 se realiza la elección de una nueva junta directiva. En dicho proceso participan dos papeletas, una encabezada por Blear y que defiende la modernización de los puertos sin la privatización de los mismos; y la otra encabezada por Brenes, y a fin a la propuesta de la administración Arias Sánchez y que sigue siendo impulsada por la Presidenta Laura Chinchilla, la cual asumió la Presidencia de la República el 8 de mayo del 2010. En dicho proceso resulta vencedor la papeleta encabezada por Blear, y la junta directiva electa se compromete a impulsar un proceso de modernización y a combatir la concesión de los puertos (Vizcaíno, 2011).

\section{Método de análisis}

Para analizar la información se recurrió al análisis de contenido, la cual consiste en lo siguiente: "(...) una técnica de investigación destinada a formular, a partir de ciertos datos, inferencias reproducibles y válidas que puedan aplicarse a su contexto" (Krippendorff, 1997 , p. 28). Para la realización de conteo e identificación de las palabras más utilizadas en las noticias recopiladas se utilizó el programa informático Wordsmith, el cual es un programa informático que permite procesar grandes cantidades de texto con el propósito de obtener listas de palabras (listado de las palabras que aparecen con mayor frecuencia dentro del texto), así como identificar palabras clave (observar en qué contexto es utilizada una determinada palabra). Segundo, una vez realizado este conteo, se procedió a agrupar las palabras en diferentes tópicos o variables, entendidos estos como constantes temáticas, sujetos o elementos a los que refieren estas palabras. Al mismo tiempo, los tópicos identificados fueron agrupados en categorías. De esta manera cada categoría se construye a partir de los elementos básicos del contenido de las noticias, y al mismo tiempo permiten observar los tópicos o temas que la constituyen según cada medio. Lo anterior permite visualizar qué temas aborda cada uno de los periódicos estudiados y qué elementos relaciona con dichos temas, para facilitar reconstruir cuál es el discurso de estos (Krippendorff, 1997, pp. 134-135). 
Tabla 1

Categorías utilizadas para el análisis de contenido de las noticias publicadas acerca del proceso de modernización de JAPDEVA por La Nación, Diario Extra, El Semanario Universidad y El País (del 8 de mayo del 2010 al 7 de mayo del 2011)

\begin{tabular}{|c|c|}
\hline Categoría & Descripción \\
\hline Actores & $\begin{array}{l}\text { Actores (individuales y colectivos) identificados por los } \\
\text { medios, así como las posiciones y argumentos expresados } \\
\text { por estos, en torno al proceso de Modernización de } \\
\text { JAPDEVA. Refiere a la información publicada en relación } \\
\text { con el proceso de concesión de los muelles }\end{array}$ \\
\hline $\begin{array}{l}\text { Proceso de } \\
\text { modernización }\end{array}$ & $\begin{array}{l}\text { Controlados por JAPDEVA, así como a distintas medidas, } \\
\text { cambios e impactos que traería este proceso para la } \\
\text { institución como para la provincia de Limón y el país en } \\
\text { general. }\end{array}$ \\
\hline & $\begin{array}{l}\text { Hace referencia al conflicto surgido a lo interno de SINTRAJAP } \\
\text { por el control de la Junta Directiva del Sindicato, así como a las } \\
\text { repercusiones de dicho conflicto podría tener en el proceso de } \\
\text { modernización de los muelles. }\end{array}$ \\
\hline Conflicto sindical & $\begin{array}{l}\text { Hace referencia al conflicto surgido a lo interno de } \\
\text { SINTRAJAP por el control de la Junta Directiva del Sindicato, } \\
\text { así como a las repercusiones de dicho conflicto podría tener } \\
\text { en el proceso de modernización de los muelles. }\end{array}$ \\
\hline
\end{tabular}

Fuente: Elaboración propia. Noviembre, 2012.

En total se identificaron tres categorías: actores, proceso de modernización y conflicto sindical (ver tabla 1). Debe señalarse que necesariamente no todas las categorías tienen la misma relevancia en el contenido de los medios estudiados; lo anterior es importante para tratar de visualizar las diferencias en el discurso sostenido por cada medio a la hora de presentar la información referente al proceso de privatización de los puertos administrados por JAPDEVA. En cuanto al corpus analizado, la tabla 2 muestra el número de noticias publicadas en los medios seleccionados, durante el periodo de estudio, relacionadas con el proceso de concesión de los puertos de JAPDEVA y el conflicto sindical generado en torno a este. 
Tabla 2

Noticiadas publicadas relacionados con la concesión de los puertos de JAPDEVA del 8 mayo del 2010 al 7 de mayo del 2011 (en números absolutos)

\begin{tabular}{lccccc}
\hline & Diario Extra & La Nación & El Semanario & El País & Total \\
\hline Mayo 2010 & 3 & 1 & 0 & 7 & $\mathbf{4}$ \\
Junio 2010 & 2 & 2 & 0 & 5 & $\mathbf{4}$ \\
Julio 2010 & 1 & 0 & 0 & 2 & $\mathbf{1}$ \\
Agosto 2010 & 8 & 11 & 3 & 9 & $\mathbf{2 2}$ \\
Septiembre 2010 & 7 & 2 & 3 & 12 & $\mathbf{1 2}$ \\
Octubre 2010 & 2 & 0 & 0 & 0 & $\mathbf{2}$ \\
Noviembre 2010 & 3 & 0 & 0 & 0 & $\mathbf{3}$ \\
Diciembre 2010 & 5 & 3 & 1 & 4 & $\mathbf{9}$ \\
Enero 2011 & 18 & 14 & 2 & 6 & $\mathbf{3 4}$ \\
Febrero 2011 & 3 & 3 & 1 & 5 & $\mathbf{7}$ \\
Marzo 2011 & 9 & 9 & 1 & 8 & $\mathbf{1 9}$ \\
Abril 2011 & 3 & 2 & 1 & 3 & $\mathbf{6}$ \\
Mayo 2011 & 0 & 0 & 0 & 1 & $\mathbf{0}$ \\
Total & $\mathbf{6 4}$ & $\mathbf{4 7}$ & $\mathbf{1 2}$ & $\mathbf{6 2}$ & $\mathbf{1 2 3}$ \\
\hline
\end{tabular}

Fuente: Elaboración propia, enero, 2012, a partir de la información encontrada en Diario Extra, La Nación, El País y el Semanario Universidad, entre el 8 de mayo del 2010 y el 7 de mayo del 2011. Enero, 2012.

\section{La visión de los participantes en el conflicto según los medios}

La categoría más importante presente en el contenido de las noticias analizadas es la de actores, en esta hay tres actores que destacan: JAPDEVA, SINTRAJAP y los trabajadores de JAPDEVA. Sobre las menciones a JAPDEVA los medios tienden a señalar cuál sería el impacto y el rol de la organización tras el proceso de concesión y privatización de los puertos, en lo cual hay opiniones dividas, ya que mientras La Nación publica noticias en las que se indica que JAPDEVA no se verá afectada tras la realización de dicho proceso, y más bien obtendrá mayor cantidad de recursos para invertir en la región (Loaiza, 2010a, 2010b); el Semanario Universidad y El País señalan como el mismo proceso puede ocasionar el debilitamiento e incluso la desaparición de JAPDEVA (Elpais.cr, 2010c, 2010e y Araya, 2010a). Se debe indicar que el proceso de concesión no implicaba la desaparición de JAPDEVA, sino que esta se convertiría en la operadora portuaria y continuaría siendo la dueña de los puertos concesionados.

En cuanto a SINTRAJAP como actor es necesario aclarar que durante el periodo de estudio existen "dos SINTRAJAP", cada uno defiende una posición opuesta respecto al proceso de concesión. Lo anterior sucede debido a, como se consignó anteriormente, la junta directiva del SINTRAJAP dirigida por Ronaldo Blear es destituida de forma ilegal, y es reemplazada por otra directiva encabezada por Douglas Brenes (como se observa en el cuadro 1, tanto Ronaldo Blear como Douglas Brenes son actores destacados en el contenido de los medios). Esta situación motiva al grupo de Blear, a la ANEP y al Partido Frente Amplio (FA) a presentar un recurso de amparo ante la Sala Constitucional, el cual 
es acogido y fallado a favor de los demandantes, por lo que es restituida la junta directiva anterior presidida por Ronaldo Blear.

Por consiguiente, el SINTRAJAP presidio por Blear es mostrado por los medios de comunicación como dispuesto a detener el proceso de concesión (Mata, 2010c; Araya, 2011d; Castro, G, 2010b y elpais.cr, 2010j), sin embargo, al mismo tiempo se indica que está anuente a dialogar con el Poder Ejecutivo para proponer el desarrollo de un proceso de modernización de los puertos (Mata, 2010c; DPA, 2010 y Espinoza, 2010d), el cual no implique ceder el control de los mismos a compañías privadas.

Si se hace reestructuración va a ser a través de medidas como -por ejemploque si alguien se pensiona, la plaza quede congelada, precisó. "Nosotros no tenemos ningún problema con el tema de la reestructuración y con analizarlo seriamente; pero, no vamos a estar haciendo cosas aisladas", dijo Castillo, tras anunciar que el sindicato presentaría el miércoles 9 de marzo -durante una nueva reunión en el Ministerio de Trabajo- una propuesta de modernización portuaria integral, a diferencia de la que presentó el Gobierno (Araya, 2011b).

EI SINTRAJAP presidido por Brenes no solo manifiesta, según los medios, una posición favorable al proceso de concesión (Loaiza, 2011b y elpais.cr, 2010c), sino que incluso es identificado como un sindicato controlado por la administración Chinchilla Miranda (Loaiza, 2011a y elpais.cr, 2010c). 


\section{Tabla 3}

Tópicos que relacionados con la categoría "Actores" en el contenido de las noticias relacionadas con el conflicto de privatización de JAPDEVA. Del 8 de mayo del 2010 al 7 de mayo del 2011. (Por medio de comunicación)

\begin{tabular}{|c|c|c|c|c|c|c|c|c|}
\hline \multirow{2}{*}{ Tópicos } & \multicolumn{2}{|c|}{ Diario Extra } & \multicolumn{2}{|c|}{ La Nación } & \multicolumn{2}{|c|}{$\begin{array}{l}\text { Semanario } \\
\text { Universidad }\end{array}$} & \multicolumn{2}{|c|}{ El País } \\
\hline & Palabras & Frecuencia & Palabras & Frecuencia & Palabras & $\begin{array}{c}\text { Frecuenci } \\
\mathbf{a}\end{array}$ & Palabras & Frecuencia \\
\hline \multirow{3}{*}{ JAPDEVA } & JAPDEVA & 208 & JAPDEVA & 165 & JAPDEVA & 93 & JAPDEVA & 178 \\
\hline & Administración & 55 & & & $\begin{array}{l}\text { Administra } \\
\text { ción }\end{array}$ & 33 & $\begin{array}{c}\text { Administració } \\
\mathrm{n}\end{array}$ & 60 \\
\hline & $\begin{array}{c}\text { Junta de } \\
\text { Administración } \\
\text { Portuaria }\end{array}$ & 30 & & & $\begin{array}{l}\text { Junta de } \\
\text { Administra } \\
\text { ción } \\
\text { Portuaria }\end{array}$ & & $\begin{array}{c}\text { Junta de } \\
\text { Administració } \\
\text { n Portuaria }\end{array}$ & 58 \\
\hline \multirow[b]{2}{*}{$\begin{array}{l}\text { Trabajador } \\
\text { es de } \\
\text { JAPDEVA }\end{array}$} & Trabajadores & 166 & $\begin{array}{c}\text { Trabajadore } \\
\mathrm{s}\end{array}$ & 58 & $\begin{array}{l}\text { Trabajador } \\
\text { es }\end{array}$ & 39 & Trabajadores & 131 \\
\hline & $\begin{array}{l}\text { Asamblea (de } \\
\text { Trabajadores) }\end{array}$ & 29 & Empleados & 22 & $\begin{array}{l}\text { Asamblea } \\
\text { (de } \\
\text { Trabajador } \\
\text { es) }\end{array}$ & & $\begin{array}{l}\text { Asamblea (de } \\
\text { Trabajadores) }\end{array}$ & 43 \\
\hline \multirow{3}{*}{$\begin{array}{l}\text { Sindicato } \\
\text { de } \\
\text { Trabajador } \\
\text { es de } \\
\text { JAPDEVA }\end{array}$} & SINTRAJAP & 143 & Sindicato & 95 & $\begin{array}{c}\text { SINTRAJA } \\
\mathrm{P}\end{array}$ & 28 & SINTRAJAP & \\
\hline & $\begin{array}{l}\text { Junta Directiva } \\
\text { del Sindicato }\end{array}$ & 21 & SINTRAJAP & 34 & $\begin{array}{c}\text { Junta } \\
\text { Directiva } \\
\text { del } \\
\text { Sindicato }\end{array}$ & 27 & $\begin{array}{c}\text { Junta } \\
\text { Directiva del } \\
\text { Sindicato }\end{array}$ & 73 \\
\hline & Sindicato & 71 & Sindicalistas & 22 & Sindicato & 45 & Sindicato & 108 \\
\hline $\begin{array}{l}\text { Ronaldo } \\
\text { Blear }\end{array}$ & Blear & 53 & Blear & 82 & & & Blear & 64 \\
\hline
\end{tabular}




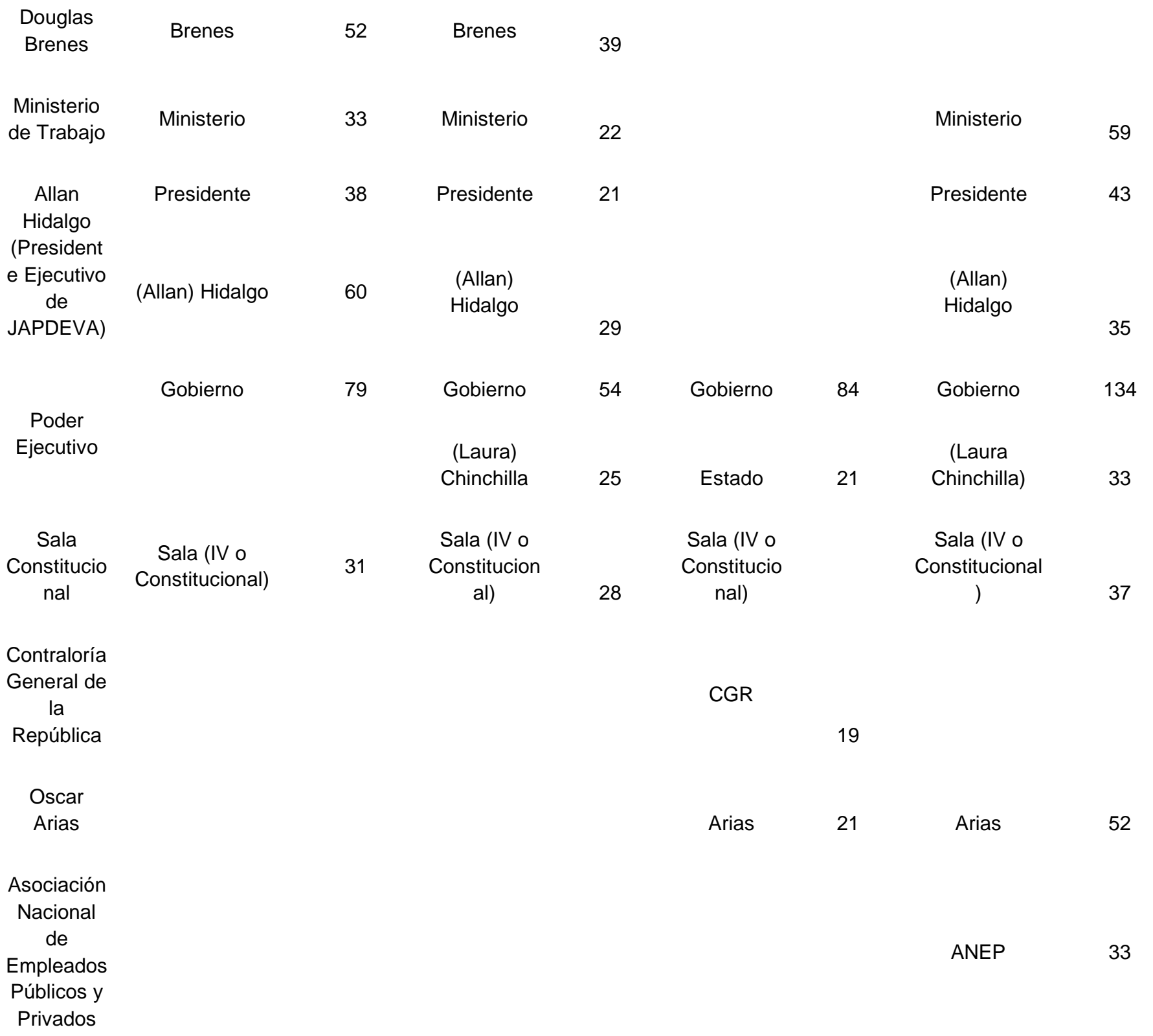

Fuente: Construcción propia, a partir de información encontrada en La Nación, el Semanario Universidad, El País y Diario Extra. Mayo, 2012

En cuanto a los trabajadores de JAPDEVA como actores, hay que destacar que existen posiciones encontradas de los medios con respecto a los estos. La Nación y Diario Extra tienden a resaltar que los problemas por los que pasa JAPDEVA se deben a las prestaciones y derechos que tienen los trabajadores de dicha institución (Loaiza, 2010a; 2010e, 2011c; Morris, G., 2011a y 2011b), por lo que, se proyecta una

José A. Díaz González

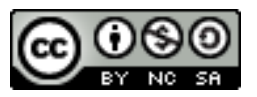


imagen de los trabajadores en donde quienes están dañando a dicha institución e impiden que esta cumpla con su objetivo de promover el desarrollo económico y social de la región Caribe costarricense.

En contraste, El País y el Semanario Universidad presentan una imagen de los trabajadores como defensores de JAPDEVA y enfatizan que estos se encuentran dispuestos a luchar tanto por la institución, como para detener el proceso de concesión que consideran negativo para la institución (Araya, 2011c; Elpais.cr, 2011a y 2011b); sin embargo, debe aclararse que estos medios tienden a presentar las acciones y opiniones de los trabajadores canalizadas a través de SINTRAJAP, de tal manera que invisibilizan las acciones de los trabajadores como colectivo fuera de dicha organización. Al mismo tiempo, los trabajadores son presentados como víctimas del proceso de concesión impulsado por el gobierno, ya que este pone en peligro sus puestos de trabajo (Araya, 2011d).

Alvarado, Álvarez y Solano (2012) han señalado que la dirigencia sindical de SINTRAJAP ha marginado la participación de las y los trabajadores; lo que ha provocado el favorecimiento de acciones aisladas que poco han contribuido la lucha de este grupo; esto puede visualizarse de forma palpable en el contenido mediático anteriormente descrito. De esta forma, el sindicato como actor colectivo se ve reducido al accionar de unas pocas personas que conforman su cúpula, mientras que el resto de sus miembros son apenas tomados en cuenta e identificados en el discurso mediático.

Otro actor relevante en el contenido de las noticias analizadas en el Ministerio de Trabajo y Seguridad Social (MTSS). Debido a que, según la legislación costarricense, el MTSS es el encargado de regular lo concerniente a las organizaciones sindicales el rol que juega este -en general- en la información presentada por los distintos medios estudiados es el de legitimar los cambios en la dirigencia de SINTRAJAP (Araya, 2010c; 2010d; Loaiza, 2010d). Es relevante como los medios le otorgan al MTSS un rol pasivo, ya que sin importar los conflictos reportados tanto dentro de SINTRAJAP como entre grupos de trabajadores, la administración de JAPDEVA y el gobierno, no se presenta que esta institución actué directamente en el intento de buscar una solución, sino que simplemente toma un papel de mediador y fiscalizador; aunque al mismo tiempo manifiesta una posición más favorable a los intereses del gobierno que a la de SINTRAJAP:

Sandra Piszk sí sostuvo que el Ministerio de Trabajo solo será mediador en las próximas reuniones. Eso sí, aclaró que no permitirán imposiciones de Ronaldo Blear, secretario general del sindicato, en la Junta de Administración Portuaria y de Desarrollo Económico de la Vertiente Atlántico. (Loaiza, 2010d)

El presidente de JAPDEVA, Allan Hidalgo, es también un actor relevante. La posición de Hidalgo retratada por los medios muestra que JAPDEVA no puede continuar funcionando adecuadamente de la forma en que lo ha venido haciendo; y es por eso que defiende el proceso de concesión de los puertos como una forma de garantizar que JAPDEVA obtenga los recursos necesarios para invertir en la región (Mata y Loaiza, 2010; Loaiza, 
2010c; Morris G, 2010c y Elpais.cr, 2010j). Asimismo, Hidalgo mantiene la tesis que es responsabilidad de los empleados de JAPDEVA su actual situación debido al abuso que estos hacen de sus derechos (Loaiza, 2010e y Morris G, 2011a).

El Poder Ejecutivo es identificado por todos los periódicos como el principal promotor del proceso de concesión (Araya, 2010d; Espinoza, 2010d y Elpais.cr, 2010j). Además, La Nación y El País destacan el papel que Laura Chinchilla realiza como presidenta de la República para llevar a cabo dicho proyecto. De tal manera, los medios enfatizan que el accionar de la administración Chinchilla Miranda sobre este tema es un continuismo de lo establecido en la administración Arias Sánchez; al mismo tiempo que señalan que los problemas generados por la propuesta de concesionar los puertos son producto del gobierno de Oscar Arias: "El culpable de esto es el Ejecutivo que era el gobierno de los Arias y que ahora, con actores similares, siguen con una participación activa en este proceso, como el ministro de Transportes, finalizó Cubero" (Mata, 2010a).

Además, en las noticias publicadas, los grupos de trabajadores tanto a favor como en contra de la concesión reprochan la actuación de la presidenta Laura Chinchilla. El primer grupo le reclama que la falta de una mayor intervención del gobierno provocó que perdieran el control del SINTRAJAP (Murillo, 2011); mientras que el grupo de trabajadores contrarios al proceso de concesión le reclama que el diálogo con el Poder Ejecutivo no avanza y que el gobierno no muestra interés de invertir en un proceso de modernización de los puertos de JAPDEVA que no implique su traslado al control de una empresa privada (Araya, 2010b; Vizcaíno, 2011 y Morris G, 2011c). De esta manera, los medios retratan a Laura Chinchilla como una figura a la que apelan distintas partes involucradas, incluso con intereses diametralmente opuestos, sin recibir ninguna una respuesta satisfactoria.

El último actor que es importante en el contenido de todos los medios es la Sala Constitucional, la cual es retratada como el ente al que se acude para solucionar un conflicto, en este caso tanto la legalidad de la junta directiva de SINTRAJAP encabezada por Douglas Brenes, como el proceso de concesión de los puertos (Redacción, 2010; Loaiza, 2011d; Ruíz, 2010; Elpais.cr, 2010f y Araya, 2011c). Al mismo tiempo, diferentes actores mencionan que es la Sala Constitucional la única autoridad competente para frenar el proceso de concesión de los puertos (Araya, 2010c; Morris G, 2010b).

\section{La imagen del proceso de modernización}

La segunda categoría encontrada en el contenido de los medios es el "proceso de modernización", el cual se relaciona con las propuestas realizadas por los distintos actores sobre cambios que deben ejecutarse en relación con los puertos controlados por JAPDEVA para mejorar su función (ver Tabla 4). Esta categoría está constituida por dos tópicos: el primero, la Concesión Portuaria que engloba las posiciones (a favor y en contra) sobre el proceso de concesión de los puertos que impulsa el gobierno. En este tópico los medios se dividen en dos posiciones; por un lado, La Nación y Diario Extra, los cuales si bien no manifiestan en sus noticias estar explícitamente a favor del proceso de concesión impulsada por el gobierno (llamado más específicamente de 
gestión interesada) dan un detallado seguimiento al proceso y destacan como avanza el mismo (Castro G, 2010a; Castro J, 2010; Espinoza, 2010d, Morris G, 2010b; Loaiza, 2010a; Mata, 2010c; Loaiza, 2010c; Mata, 2010c y 2010d).

Tabla 4.

Tópicos relacionados con la categoría "Proceso de modernización" en el contenido de las noticias relacionadas al proceso de modernización de JAPDEVA. Del 8 de mayo del 2010 al 7 de mayo del 2011. (Por medio de comunicación)

\begin{tabular}{|c|c|c|c|c|c|c|c|c|}
\hline \multirow{2}{*}{ Tópicos } & \multicolumn{2}{|c|}{ Diario Extra } & \multicolumn{2}{|c|}{ La Nación } & \multicolumn{2}{|c|}{ Semanario Universidad } & \multicolumn{2}{|c|}{ El País } \\
\hline & Palabras & Frecuencia & Palabras & Frecuencia & Palabras & Frecuencia & Palabras & Frecuencia \\
\hline \multirow{7}{*}{$\begin{array}{l}\text { Concesión } \\
\text { Portuaria }\end{array}$} & Concesión & 83 & Concesión & 65 & Concesión & 84 & Concesión & 53 \\
\hline & Muelles & 81 & Muelles & 47 & Muelles & 28 & Muelles & \\
\hline & Portuaria & 81 & Portuaria & 50 & Portuaria & 31 & Portuaria & 56 \\
\hline & Modernización & 63 & Modernización & 32 & Modernización & 29 & Servicios & 34 \\
\hline & Puertos & 54 & Puertos & 25 & Puertos & 48 & & \\
\hline & Servicios & 31 & & & Licitación & 26 & & \\
\hline & Gestión & 29 & & & Cartel & 32 & & \\
\hline $\begin{array}{l}\text { Convención } \\
\text { Colectiva }\end{array}$ & $\begin{array}{l}\text { Convención } \\
\text { Colectiva }\end{array}$ & 78 & $\begin{array}{l}\text { Convención } \\
\text { Colectiva }\end{array}$ & 33 & & & & \\
\hline
\end{tabular}

Fuente: Construcción propia, a partir de información encontrada en La Nación, el Semanario Universidad, El País y Diario Extra. Mayo, 2012.

Asimismo, La Nación y Diario Extra tienden a destacar los beneficios que acarreará el proceso de concesión a la zona del Caribe, en especial, hacen énfasis en el aumento de recursos que tendrá JAPDEVA para invertir en el desarrollo social de la zona: "La cantidad de dinero que se destina a inversión social en el Caribe costarricense se triplicaría cuando la operación de los muelles de Limón y Moín, y de una terminal para portacontenedores se entregue a operadores privados" (Loaiza, 2010a). 
También, estos dos medios enfatizan el tema de la Convención Colectiva existente como uno de los puntos necesarios por tratar para alcanzar el proceso de modernización (ver tabla 4). Sobre este punto La Nación y Diario Extra mencionan que la convención colectiva es un obstáculo para el buen funcionamiento de JAPDEVA, ya que proporciona una serie de beneficios -según su criterio- excesivos a los trabajadores de la institución (Mata, 2010b; Loaiza, 2010c; Espinoza, 2010a; 2010b, 2010c y Carvajal, 2010).

Por otra parte, El País y el Semanario Universidad al mencionar en su contenido el proceso de modernización lo hacen destacando la posición de SINTRAJAP (Elpais.cr, 2010f, 2010i; DPA, 2010; Araya, 2010a y 2010c), quien argumenta que es posible llevar a cabo un proceso de modernización para mejorar la eficiencia de los puertos sin necesidad de privatizarlos.

Las y los trabajadores de JAPDEVA quieren seguir sirviéndole al país y al pueblo de Limón y están a la espera de que el gobierno les presente las propuestas de modernización, que al menos incluyan compra de equipo, ampliación de las instalaciones portuarias y medidas correctivas de la situación de las tarifas y los ingresos", apuntó la organización en un documento distribuido a los trabajadores (Elpais.cr, 2011a).

Por lo tanto, se percibe un enfrentamiento sobre el tipo y la forma de modernización que debe llevarse a cabo en los puertos de JAPDEVA en el contenido de los medios analizados. Por un lado, La Nación y Diario Extra respaldan la visión del gobierno, la cual indica que es necesario privatizar los puertos para que estos sean eficientes, al mismo tiempo que identifican a los propios trabajadores de SINTRAJAP como un obstáculo para alcanzar dicho objetivo. Por otro lado, el Semanario Universidad y El País dan mayor importancia a la posición de los trabajadores, quienes defienden que puede realizarse un proceso de modernización de los puertos sin necesidad de su concesión, a la vez que identifican como responsables de los problemas por los que atraviesa JAPDEVA al gobierno, en especial a la administración Arias Sánchez (20062010), de propiciar la crisis de la institución para impulsar la privatización de la misma:

Los responsables del Gobierno pasado "fabricaron" una crisis con los puertos de Limón para hacerle creer a la opinión pública que era necesaria la concesión, pero ese y los otros planes de modernización anunciado no caminan porque están manejados por gente que desconoce la realidad de la provincia. (Araya, 2010a).

\section{Conflicto por el control de la Junta Directiva de SINTRAJAP}

Uno de los temas abordados por los medios analizados y al que dan un seguimiento constante (excepto por el Semanario Universidad), es el conflicto en torno a la conformación de la junta directiva del SINTRAJAP y el proceso electoral llevado a cabo para elegir la misma en enero del 2011. Como se observa en la tabla 2, la información publicada por los medios pueden agruparse en dos momentos; el primero, las noticias relacionadas con la destitución y restitución de la junta directiva de SINTRAJAP presidida 
por Rolando Blear. En este caso, por ejemplo, Diario Extra muestra como el conflicto existente entre el grupo de Blear y el grupo dirigido por Douglas Brenes llega a tal punto que el primero se niega a entregar el edificio del sindicato al segundo (ya que no reconoce como legítima su destitución), lo cual provoca que sea necesario la intervención de la policía para desalojarlos.

\section{Tabla 5.}

Titulares publicados por La Nación, Diario Extra y El País sobre la elección de la Junta Directiva de SINTRAJAP (8 de mayo, 2010 al 7 de mayo, 2011)

\begin{tabular}{|c|c|c|c|c|c|}
\hline \multicolumn{2}{|c|}{ La Nación } & \multicolumn{2}{|c|}{ Diario Extra } & \multicolumn{2}{|c|}{ El País } \\
\hline Titular & Fecha & Titular & Fecha & Titular & Fecha \\
\hline $\begin{array}{l}\text { Se caldea la } \\
\text { lucha por la } \\
\text { junta sindical } \\
\text { de Japdeva }\end{array}$ & $12 / 01 / 2011$ & $\begin{array}{lr}\text { Ministerio } & \text { de } \\
\text { trabajo } & \text { avala } \\
\text { nueva } & \\
\text { convención } & \text { de } \\
\text { japdeva } & \end{array}$ & $12 / 05 / 2010$ & $\begin{array}{lr}\text { Frente } & \text { Amplio } \\
\text { impugnó } & \text { nueva } \\
\text { Convención } & \\
\text { Colectiva } & \text { de } \\
\text { JAPDEVA en } & \text { Sala IV }\end{array}$ & $14 / 06 / 2010$ \\
\hline $\begin{array}{l}\text { Sindicato de } \\
\text { puertos } \\
\text { recobró poder } \\
\text { por error de la } \\
\text { oposición }\end{array}$ & $17 / 01 / 2011$ & $\begin{array}{l}\text { Ex sindicalistas } \\
\text { de japdeva no } \\
\text { abandonarán } \\
\text { edificio }\end{array}$ & $25 / 05 / 2010$ & $\begin{array}{ll}\text { Denuncian falta } & \text { de } \\
\text { transparencia } & \text { en } \\
\text { privatización } & \text { de } \\
\text { puertos de Limón } & \end{array}$ & $18 / 06 / 2010$ \\
\hline $\begin{array}{l}22 \text { sindicalistas } \\
\text { con derecho al } \\
\text { voto no } \\
\text { trabajan para } \\
\text { Japdeva }\end{array}$ & $18 / 01 / 2011$ & $\begin{array}{l}\text { Policía } \\
\text { sintrajap }\end{array}$ & $27 / 05 / 2010$ & $\begin{array}{l}\text { Dirigencia legítima } \\
\text { de SINTRAJAP } \\
\text { solicita reunión con } \\
\text { Presidenta Chinchilla }\end{array}$ & 08/07/2010 \\
\hline $\begin{array}{ll}\begin{array}{l}\text { Sindicato } \\
\text { Japdeva }\end{array} & \text { de } \\
\text { defraudó } & \text { a } \\
\text { Chinchilla } & \end{array}$ & $20 / 01 / 2011$ & $\begin{array}{l}\text { Sala cuarta } \\
\text { restituye dirigente } \\
\text { de sintrajap }\end{array}$ & $26 / 08 / 2010$ & $\begin{array}{l}\text { Sindicato de EEUU } \\
\text { demanda al gobierno } \\
\text { de Costa Rica por } \\
\text { violaciones laborales }\end{array}$ & $23 / 07 / 2010$ \\
\hline $\begin{array}{l}\text { Defensoría } \\
\text { será } \\
\text { observadora } \\
\text { en elección } \\
\text { sindical de } \\
\text { Japdeva }\end{array}$ & $20 / 01 / 2011$ & $\begin{array}{l}\text { ¿Trabajadores de } \\
\text { japdeva sin } \\
\text { convención } \\
\text { colectiva? }\end{array}$ & 28/08/2010 & $\begin{array}{l}\text { Directiva legal de } \\
\text { SINTRAJAP celebra } \\
\text { reinstalación de Sala } \\
\text { Constitucional }\end{array}$ & $26 / 08 / 2010$ \\
\hline $\begin{array}{l}\text { Trabajo niega } \\
\text { obligación de }\end{array}$ & $20 / 01 / 2011$ & Sintrajap & $04 / 01 / 2011$ & $\begin{array}{l}\text { Se derrumba inmoral } \\
\text { mordida de los } 80 \text { mil }\end{array}$ & $26 / 08 / 2010$ \\
\hline
\end{tabular}




\begin{tabular}{|c|c|c|c|c|c|}
\hline $\begin{array}{l}\text { verificar padrón } \\
\text { electoral de } \\
\text { Sintrajap }\end{array}$ & & observadores & & millones de colones & \\
\hline $\begin{array}{l}\text { Limón agitado } \\
\text { por elección } \\
\text { sindical de } \\
\text { mañana }\end{array}$ & $20 / 01 / 2011$ & $\begin{array}{l}\text { Sintrajap salió } \\
\text { respondón }\end{array}$ & 05/01/2011 & $\begin{array}{l}\text { Frente Amplio } \\
\text { celebra restitución de } \\
\text { legalidad en } \\
\text { SINTRAJAP }\end{array}$ & $27 / 08 / 2010$ \\
\hline $\begin{array}{l}\text { Sindicalistas } \\
\text { acuden a } \\
\text { asamblea que } \\
\text { decidirá futuro } \\
\text { de puertos } \\
\text { limonenses }\end{array}$ & $21 / 01 / 2011$ & $\begin{array}{l}\text { Policías velarán } \\
\text { por libre tránsito } \\
\text { en limón }\end{array}$ & $10 / 01 / 2011$ & $\begin{array}{l}\text { Fracciones de } \\
\text { oposición llaman a } \\
\text { negociación por } \\
\text { puertos de Limón }\end{array}$ & $27 / 08 / 2010$ \\
\hline $\begin{array}{l}\text { Primer } \\
\text { encontronazo } \\
\text { en Asamblea } \\
\text { de Sintrajap }\end{array}$ & $21 / 01 / 2011$ & $\begin{array}{lr}\text { Hoy primera } \\
\text { convocatoria } & \text { de } \\
\text { asamblea } & \text { de } \\
\text { sintrajap } & \end{array}$ & 14/01/2011 & $\begin{array}{l}\text { Si concesionaron la } \\
\text { Patria, pueden } \\
\text { concesionar } \\
\text { cualquier cosa }\end{array}$ & $28 / 08 / 2010$ \\
\hline $\begin{array}{l}\text { Sindicalistas } \\
\text { eligen a cúpula } \\
\text { opuesta a } \\
\text { concesión } \\
\text { portuaria }\end{array}$ & $22 / 01 / 2011$ & $\begin{array}{l}\text { Chinchilla pide } \\
\text { respaldo para } \\
\text { concesión } \\
\text { portuaria }\end{array}$ & $20 / 01 / 2011$ & $\begin{array}{l}\text { Chinchilla trata de } \\
\text { remover obstáculos } \\
\text { para modernizar } \\
\text { puertos }\end{array}$ & $31 / 08 / 2010$ \\
\hline $\begin{array}{l}\text { 'Se rechazó la } \\
\text { concesión' }\end{array}$ & $22 / 01 / 2011$ & $\begin{array}{l}\text { Japdevianos } \\
\text { dijeron ¡no! A la } \\
\text { concesión }\end{array}$ & $22 / 01 / 2011$ & $\begin{array}{l}\text { Elección en } \\
\text { SINTRAJAP }\end{array}$ & $18 / 01 / 2011$ \\
\hline $\begin{array}{l}\text { Gobierno } \\
\text { insiste en } \\
\text { concesión, } \\
\text { pero no sabe } \\
\text { cómo } \\
\text { alcanzarla }\end{array}$ & $22 / 01 / 2011$ & $\begin{array}{l}\text { Cúpula de } \\
\text { sintrajap solicitó } \\
\text { inscripción }\end{array}$ & $31 / 01 / 2011$ & $\begin{array}{l}\text { Defensoría de los } \\
\text { Habitantes observará } \\
\text { elección del sindicato } \\
\text { de Japdeva }\end{array}$ & $21 / 01 / 2011$ \\
\hline $\begin{array}{l}\text { Gasto de \$55 } \\
\text { millones } \\
\text { enfrentó a } \\
\text { sindicalistas }\end{array}$ & $22 / 01 / 2011$ & & & $\begin{array}{lr}\text { Elecciones } & \text { de } \\
\text { directiva } & \text { en } \\
\text { SINTRAJAP } & \text { con } \\
\text { presiones } & \\
\text { gubernamentales } & \end{array}$ & $21 / 01 / 2011$ \\
\hline $\begin{array}{l}\text { Grupo } \\
\text { perdedor en } \\
\text { Japdeva culpa } \\
\text { al Gobierno }\end{array}$ & $23 / 01 / 2011$ & & & $\begin{array}{l}\text { Trabajadores de } \\
\text { Japdeva dijeron NO } \\
\text { a la privatización de } \\
\text { los puertos de Limón }\end{array}$ & $22 / 01 / 2011$ \\
\hline
\end{tabular}




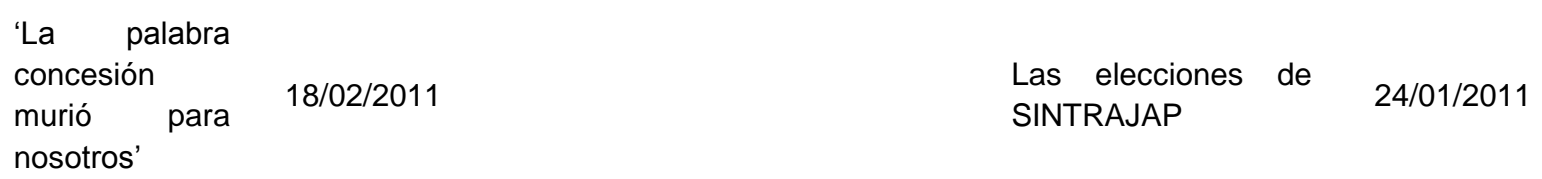

Fuente: Elaboración propia, a partir de la información encontrada en La Nación, Diario Extra y El País, entre el 8 de mayo del 2010 al 7 de mayo del 2011. Junio, 2012.

Además, El País muestra como un actor relevante en su contenido al FA, el cual interviene en favor del grupo liderado por Blear mediante la toma de acciones con el propósito de frenar el proceso de negociación de una nueva convención colectiva llevada a cabo por la junta directiva liderada por Brenes:

El diputado del Partido Frente Amplio, José María Villalta, presentó una acción de inconstitucionalidad contra lo que considera "un chantaje, un soborno absolutamente ilegal y que dejaron plasmado en una reforma a la Convención Colectiva del Sindicato SINTRAJAP con la complicidad de una junta directiva espuria. (Elpais.cr, 2010b).

En cuanto a la restitución de la junta directiva presidida por Blear, los medios son claros en manifestar que esta se da debido a una resolución de la Sala Constitucional que declara ilegal la forma en que fueron removidos de su cargo. A pesar de que el grupo liderado por Brenes estaba a favor de la concesión de los puertos, y La Nación y Diario Extra también se posicionan en favor de esta propuesta; no expresan mayor crítica por la decisión de la Sala, la cual corresponde a una decisión adoptada tras seguir la normativa y los pasos institucionalizados, y en el discurso de ambos medios el respeto a la institucionalidad es fundamental (

Este respeto a la institucionalidad en el discurso de los medios se puede observar, más claramente, en el segundo momento identificado en relación con la información publicada sobre la junta directiva de SINTRAJAP, y corresponde al seguimiento que hacen los medios a las elecciones que se llevan a cabo en enero del 2011 para elegir la junta directiva de la organización. Esto se muestra en el interés de los medios en informar acerca de cómo se realizará dicho proceso electoral, al tiempo que manifiestan acerca de la presencia tanto de un observar externo (la Defensoría de los Habitantes) y la participación de la policía para "resguardar el orden" en la ciudad de Limón, como una garantía de la transparencia del proceso.

Ciertamente, la preocupación por el proceso de elección se debe a que, según lo presentan los medios, este definirá el proceso de modernización que se llevará a cabo en JAPDEVA; ya que la elección se realizó entre el grupo de Blear (contra la concesión) y el grupo de Brenes (en favor de la concesión). El resultado del proceso le da la victoria al grupo de Blear y, a pesar de algunas acusaciones presentadas por Douglas Brenes sobre la legalidad de la candidatura de Blear -las cuales no fructificaron-, los medios no reportan que ocurriera ninguna anormalidad o indicio de fraude en la elección. 
Dado que los medios dieron seguimiento a los controles necesarios para asegurar la transparencia del proceso donde aceptan como legítima la elección del grupo de Blear, esto a pesar de ser contrario a la visión que defienden (caso de La Nación y Diario Extra). Sin embargo, La Nación hace eco de las declaraciones del grupo perdedor, el cual culpa al gobierno por "no intervenir" lo suficiente, de tal manera que provoca así su derrota:

Doña Laura (Chinchilla) no se pronunció hasta dos días antes de la elección y muchos miembros del sindicato dudaban de la indemnización ofrecida (para aceptar la concesión). El apoyo que tuvimos fue nulo y eso nos obligó a trabajar con las uñas". Esta fue la reflexión que dio ayer Douglas Brenes, quién el viernes no obtuvo votos suficientes para desplazar a Ronaldo Blear... A pesar de la amplia diferencia de votos, Douglas Brenes dijo que el panorama habría sido diferente si Chinchilla y su gabinete hubieran sido más claros en su mensaje sobre la intención de pagar una "indemnización" cercana a los $\$ 137$ millones, a cambio de acceder a dejar los puertos en manos privadas. (Murillo, 2011).

Lo anterior es significativo, ya que, como otros medios habían informado, se denunciaba que había existido una abierta intromisión de la administración Arias Sánchez en tanto en JAPDEVA como en SINTRAJAP para facilitar el proceso de concesión de los puertos (ANEP, 2010; Morera, 2009 y Araya, 2010a), las declaraciones de Brenes parecieran apoyar esas suposiciones, al manifestar que el proceso de privatización no era algo que los trabajadores de la_institución estaban de acuerdo, sino que requería de una intervención o incentivo por parte de la administración Chinchilla Miranda para que apoyaran dicha posición.

\section{Consideraciones finales}

Si bien los medios no controlan el pensamiento de las personas a las que dirigen su mensaje, es decir, no les indican cómo pensar; al dar énfasis y seguimiento a determinada información y minimizar o ignorar otra en sus publicaciones, inciden directamente en que la población identifique como de su interés temas específicos, en otras palabras, le indican en qué pensar (Bustos, 2011, p. 111). Asimismo, el sociólogo alemán Niklas Luhmann reconoce que los medios, de una u otra forma describen a la sociedad; así, por ejemplo, el desarrollo de las sociedades capitalistas ha llevado al predominio de los valores mercantiles en el sistema de medios de comunicación (Luhmann, 2007).

A partir de lo anterior, no es de extrañar entonces como los medios estudiados construyen dos narrativas diferentes sobre el proceso de concesión de los puertos de JAPDEVA, las cuales son incluso antagónicas, y así muestra cuál es la posición de los medios sobre dicho tema. Por un lado, se encuentran La Nación y Diario Extra, quienes toman una postura en favor del proceso de concesión, da énfasis en su contenido de que se trata de un proceso de modernización, es decir, procuran mostrar a la opinión pública que su objetivo es mejorar el funcionamiento de los puertos administrados por JAPDEVA, en donde se oculta o minimiza que el control del mismo pasaría de ser público a estar en 
manos privadas. Por otro lado, El País y el Semanario Universidad en su contenido denuncian las irregularidades y problemas que traería el proceso de privatización de los puertos, por lo que al referirse a modernización lo que señalan es la iniciativa del grupo sindical dirigido por Blear para realizar cambios e inversiones para el mejoramiento del funcionamiento de los puertos, sin que esto implique que pasen a estar en control de una empresa privada 4 .

Asimismo, el análisis del contenido de las noticias publicadas por el Semanario Universidad, La Nación, El País y Diario Extra durante el periodo de estudio muestra que estos visualizan el conflicto como el enfrentamiento de dos posiciones representadas por grupos bien definidos; el primero, en favor de la concesión de los puertos, el cual incluye al gobierno (administración Arias Sánchez y administración Chinchilla Miranda), la administración de JAPDEVA y un grupo de empleados de la institución (Grupo Mediador); y el segundo en contra de la propuesta de concesión, en el cual se encuentra SINTRAJAP, apoyado por otros sindicatos y algunos partidos políticos.

De esta manera, la imagen que presenta los medios es la del choque de estos dos grupos, por lo que dicho conflicto solo podría llegar a su final tras un acuerdo entre ambas partes. Esta imagen ignora, o al menos deja en segundo plano, que el trasfondo del conflicto se encuentra en el proceso de cambio del modelo de Estado impulsado por las políticas neoliberales promovidas en Costa Rica a partir de finales del siglo XX, y con las cuales se promueve que los Estados tengan la menor injerencia posible en las actividades productivas y dejen estas en manos del sector privado.

Además, los medios de comunicación estudiados presentan como el gobierno busca influir en las decisiones internas de SINTRAJAP, con el propósito de que la posición de este sea más conveniente para sus intereses. En este caso también se visualizan dos posiciones, por un lado la del Semanario Universidad y El País, quienes denuncian los intentos del gobierno de influir en las decisiones internas del SINTRAJAP y de buscar manipular a los trabajadores y las trabajadoras; y por el otro, La Nación y Diario Extra que no mencionan esta injerencia. Sin embargo, es posible inferir que La Nación culpa a la administración Chinchilla Miranda de no intervenir lo suficiente, al darle espacio en sus noticias a las quejas del grupo perdedor del proceso electoral interno de SINTRAJAP, liderado por Brenes, quién culpa al gobierno de su derrota.

En cuanto a la injerencia del Poder Ejecutivo en el funcionamiento de las organizaciones sindicales en Costa Rica hay que anotar que no es nueva, ya desde la década de 1980 es posible rastrear una serie de acciones adoptadas por distintas Administraciones con el propósito de reducir el espacio de maniobra de estas organizaciones, al mismo tiempo que buscan debilitarlas y desacreditarlas ante la opinión pública, con el propósito de disminuir su capacidad de influencia y acción en el espacio público (Díaz, 2010, pp. 149-

\footnotetext{
${ }^{4}$ Es necesario indicar que, en la narrativa de los medios de comunicación, tiende a ver una equiparación de término concesión con el de privatización. Por lo tanto, si bien la figura jurídica que promovía las administraciones Arias Sánchez y Chnchilla Miranda no correspondía a la privatización o venta de un bien público, la idea que termina transmitiéndose a la población es que efectivamente se estaría realizando un proceso de privatización de los puertos controlados por JAPDEVA.
} 
156). Asimismo, la injerencia, primero, de la administración Arias Sánchez y, posteriormente, de la administración Chinchilla Miranda, tanto en los procesos internos de SINTRAJAP, como en todo el proceso de búsqueda de implementación del proyecto de modernización portuaria, responde a una orquestación de la institucionalidad por parte de la élite política con el propósito de profundizar el proyecto neoliberal que ha venido impulsado en Costa Rica desde finales del siglo XX (Alvarado y otros, 2012, p. 218).

Además, es necesario señalar que en el contenido de las noticias publicadas por Diario Extra y La Nación se enfatiza en el hecho de que son los derechos de los trabajadores y trabajadoras de JAPDEVA las causas de la crisis que afronta la institución, sobre todo porque se da a entender que hay un abuso sistemático y reiterativo de los mismos. En ese sentido estos medios parecen que buscan perpetuar en la opinión pública la imagen del funcionario público como "vago" e irresponsable, cuyo abuso de sus derechos va en detrimento de la institución en la que labora y, por ende, de todo el país. Asimismo, la imagen que se presenta de SINTRAJAP es la de obstruccionista a la voluntad del gobierno, la que estos medios parecen asumir es sinónimo de la voluntad popular, lo que parece ir en la línea de reforzar la idea de que los sindicatos son obstáculos para el desarrollo de Costa Rica.

Por último, se destaca como todos los medios estudiados presentan que el conflicto se debe al choque de posturas de dos grupos bien definidos (a favor y en contra de la concesión), y no mencionan en ningún momento cuál es el parecer de la población costarricense en general sobre este tema. Esta invisibilización que se realiza del resto de la población puede facilitar, entonces, que los temas que se discutan en la agenda pública sean referentes a los intereses de esos dos grupos, lo cual excluye los intereses que pueda tener el resto de la sociedad costarricense sobre el tema, así como se facilita que se marginen de una decisión pública.

\section{Referencias}

Alvarado, A., Álvarez, M. y Solano, S. (agosto-septiembre, 2012). Puertos, autoritarismos y resistencias: El conflicto por la concesión de los puertos de Limón. Revista Reflexiones, Número Especial Jornadas de Investigación Interdisciplinarias, 211222. Recuperado de: http://www.reflexiones.fcs.ucr.ac.cr/index.php/edicionesreflexiones/numeroespecial-jornadas-interdisciplinarias-en-ciencias-sociales

Asociación Nacional de Empleados Públicos. (18 de enero de 2010). Pronunciamiento de ANEP sobre intromisión del Gobierno en Sintrajap. El Pregon.org. Recuperado de: http://ns2.elpregon.org/v2/mipregon/98-mi-opinion/2129- pronunciamiento-deanep-sobre-intromision-del-gobierno-en-sintrajap

Araya, J. L. (4 de agosto 2010a). Juan Francisco Montealegre, empresario: Gobierno fabricó crisis para permitir privatización de muelles. Semanario Universidad. Recuperado de: http://semanariouniversidad.ucr.cr/pais/juan-francisco- 
montealegre-empresario-gobierno-fabric-crisis-para-permitir-privatizacin-demuelles/

Araya, J. L. (18 de agosto 2010b). ANEP pide explicaciones a Ministro de Transportes: Preocupa que concesión portuaria eleve tarifas en Limón. Semanario Universidad. Recuperado de http://semanariouniversidad.ucr.cr/pais/anep-pide-explicaciones-aministro-de-transportes-preocupa-que-concesin-portuaria-eleve- tarifas-en-limn/

Araya, J. L. (25 de agosto 2010c). Allan Hidalgo, presidente de JAPDEVA: Aumento de tarifa portuaria de \$169 a \$256 es razonable. Semanario Universidad. Recuperado de http://semanariouniversidad.ucr.cr/pais/allan-hidalgo-presidente- de-japdevaaumento-de-tarifa-portuaria-de-169-a-256-es-razonable/

Araya, J. L. (1 de septiembre 2010d). Restitución de directiva opuesta a concesión de muelles. Semanario Universidad. Recuperado de http://semanariouniversidad.ucr.cr/pais/restitucin-de-directiva-opuesta-a-concesinde-muelles/

Araya, J. L. (25 de enero 2011a). Elección de sindicato en JAPDEVA decide concesión de puertos del Caribe. Semanario Universidad. Recuperado de: http://semanariouniversidad.ucr.cr/pais/eleccin-de-sindicato-en-japdeva-decideconcesin-de-puertos-del-caribe/

Araya, J. L. (25 de enero 2011b). Muelleros de SINTRAJAP piden inversión para superar rezago portuario. Semanario Universidad. Recuperado de http://semanariouniversidad.ucr.cr/pais/muelleros-de-sintrajap-piden-inversin- parasuperar-rezago-portuario/

Araya, J. L. (9 de marzo 2011c). Sindicato de JAPDEVA impugnará concesión de nuevo puerto en Moín. Semanario Universidad. Recuperado de http://semanariouniversidad.ucr.cr/pais/sindicato-de-japdeva-impugnar-concesinde-nuevo-puerto-en-mon/

Araya, J. L. (13 de abril 2011d).Denuncian al Gobierno por dar ventajas "ilegales" en concesión de nuevo puerto en el Caribe. Semanario Universidad. Recuperado de http://semanariouniversidad.ucr.cr/pais/denuncian-al-gobierno-por-dar- ventajasilegales-en-concesin-de-nuevo-puerto-en-el-caribe/

Bustos Mora, G. (Julio, 2011). "La mirada mediática hacia "Tierra Dominicana": una aproximación desde el Análisis Crítico del Discurso (ACD)".Rupturas 1(1). Recuperado http://investiga.uned.ac.cr/revistas/index.php/rupturas/article/view/145

Carvajal, M. (16 de agosto 2010). Puertos del Caribe abiertos los 365 días. Diario Extra. Recuperado de http://www.diarioextra.com/2010/agosto/16/nacionales03.php 
Castro, J. (25 de agosto 2010). MOPT defiende proceso de concesión de Moín. Diario Extra. Recuperado de http://www.diarioextra.com/2010/agosto/25/nacionales08.php

Castro G. C. (11 de agosto 2010a). Avanza concesión de puertos del Caribe. Diario Extra. Recuperado de http://www.diarioextra.com/2010/agosto/11/nacionales08.php

Castro G. C. (26 de agosto 2010b). Sala IV restituye a dirigente de SINTRAJAP. Diario Extra. Recuperado de http://www.diarioextra.com/2010/agosto/26/nacionales18.php

Díaz González, J. A. (2010) "Propuesta de periodización del desarrollo de las organizaciones sindicales en Costa Rica: 1932-1998." Revista de Ciencias Sociales № 128-129. Recuperado de http://revistacienciassociales.ucr.ac.cr/wpcontent/revistas/128-129/09-DIAZ.pdf

DPA. (31 de agosto 2010). Chinchilla trata de remover obstáculos para modernizar puertos. El País. Recuperado de http://www.elpais.cr/articulos.php?id=31298

ELPAIS.CR (14 de junio 2010b). Frente Amplio impugnó nueva Convención Colectiva de JAPDEVA en Sala IV. El País. Recuperado de: http://www.elpais.cr/articulos.php?id=26476

ELPAIS.CR (18 de junio 2010c). Denuncian falta de transparencia en privatización de puertos de Limón. El País. Recuperado de http://www.elpais.cr/articulos.php?id=26730

ELPAIS.CR (11 de agosto 2010e). Muelles del Caribe de Costa Rica en manos privadas serán menos competitivos. El País. Recuperado de http://www.elpais.cr/articulos.php?id=30147

ELPAIS.CR (27 de agosto 2010f). Frente Amplio celebra de legalidad en SINTRAJAP. EI País. Recuperado de http://www.elpais.cr/articulos.php?id=31071

ELPAIS.CR (27 de agosto 2010g). Sintrajab propone modernizar los puertos sin concesionarlos. El País. http://www.elpais.cr/articulos.php?id=31072

ELPAIS.CR (02 de septiembre 2010i). FA respalda modernización de puertos de Limón, sin privatizarlos. El País. Recuperado de http://www.elpais.cr/articulos.php?id=31410

ELPAIS.CR (3 de septiembre 2010j). Gobierno y SINTRAJAP negociarán futuro de puertos del Caribe de Costa Rica. El País. Recuperado de http://www.elpais.cr/articulos.php?id=31466 
ELPAIS.CR (14 de febrero 2011a). Gobierno quiere imponer privatización de puertos, denuncia SINTRAJAP. El País. Recuperado de http://www.elpais.cr/articulos.php?id=41140

ELPAIS.CR (05 de marzo 2011b). Mega puerto de Moín adjudicado con mañas y mentiras, denuncia SINTRAJAP. El País. Recuperado de http://www.elpais.cr/articulos.php?id=42223

Espinoza, M. (17 de octubre 2008). Indemnización de JAPDEVA costara entre $\$ 60$ y $\$ 80$ millones Diario Extra. Recuperado de http://www.diarioextra.com/2008/octubre/17/nacionales05.php

Espinoza, M. (11 de mayo 2010a). Ministerio de Trabajo avala nueva convención de JAPDEVA. Diario Extra. Recuperado de http://www.diarioextra.com/2010/mayo/12/nacionales02.php

Espinoza, M. (15 de junio 2010b). Acción de Inconstitucionalidad por indemnización en Japdeva. Diario Extra. Recuperado de http://www.diarioextra.com/2010/junio/15/nacionales06.php

Espinoza, M. (28 de agosto 2010c). Sindicato de JAPDEVA sin convención colectiva?. Diario Extra. Recuperado de http://www.diarioextra.com/2010/agosto/28/nacionales04.php

Espinoza, M. (31 de agosto 2010d). SINTRAJAP verá concesión de puertos con Presidenta. Diario Extra. Recuperado de http://www.diarioextra.com/2010/agosto/31/nacionales03.php

INFORMA-TICO.COM. (29 de octubre 2008). Sindicato de JAPDEVA advierte sobre pretensión de privatizar puertos. Informa-tico. Recuperado de http://www.elpregon.org/v2/costarica/economia/630-sindicato-de-japdeva-adviertesobre-pretension-de-privatizar-puertos

Krippendorff, K. (1997) Metodología de análisis de contenido. Teoría y Práctica. Barcelona, Paidós. $\quad$ http://www.planetadelibros.com/metodologia-de-analisis-de-contenidoteoria-y-practica-libro-19226.html

Loaiza, V. (10 de junio 2010a). Concesión de puertos triplicaría la inversión social en Limón. La Nación. Recuperado de http://www.nacion.com/2010-0611/EIPais/FotoVideoDestacado/EIPais2403637.aspx

Loaiza, V. (10 de junio 2010b). Japdeva administrará fondos para progreso local. La Nación. Recuperado de http://www.nacion.com/2010-0611/EIPais/Relacionados/EIPais2403651.aspx 
Loaiza, V. (29 de agosto 2010c). Japdeva proyecta números rojos en los puertos para el 2011. La Nación. Recuperado de http://www.nacion.com/2010-0830/EIPais/NotasSecundarias/EIPais2501016.aspx

Loaiza, V. (31 de agosto 2010d). Gobierno sigue sin respuestas sobre futuro de puertos. La Nación. Recuperado de http://www.nacion.com/2010-0901/EIPais/NotasSecundarias/EIPais2505600.aspx

Loaiza, V. (09 de diciembre 2010e) Ministerio Público investiga incapacidades en Japdeva. La Nación. Recuperado de http://www.nacion.com/2010-1210/EIPais/NotasSecundarias/EIPais2617920.aspx

Loaiza, V. (11 de enero 2011a) "Se caldea la lucha por la junta sindical de Japdeva. La Nación. Recuperado de http://www.nacion.com/20110112/EIPais/NotasSecundarias/EIPais2648576.aspx

Loaiza, V. (17 de enero 2011b) 22 sindicalistas con derecho al voto no trabajan para Japdeva. La Nación. Recuperado de http://www.nacion.com/2011-0118/EIPais/NotasSecundarias/EIPais2654715.aspx

Loaiza, V (21 de enero 2011c) Gobierno insiste en concesión, pero no sabe cómo alcanzarla. La Nación. Recuperado de http://www.nacion.com/2011-0122/EIPais/NotasSecundarias/EIPais2659183.aspx

Loaiza, V (16 de febrero 2011d). Sindicato de puertos recobró poder por error de la oposición. La Nación. Recuperado de http://www.nacion.com/2011-0117/EIPais/NotasSecundarias/EIPais2653572.aspx

Luhmann, N. (2007). La realidad de los medios de masas. Barcelona, Anthropos Editorial http://www.anthropos-editorial.com/DETALLE/LA-REALIDAD-DE-LOS-MEDIOSDE-MASAS-BA-040

Mata, A. (26 de agosto 2010a). Oposición celebra reinstalación de sindicalistas de Japdeva. La Nación. Recuperado de http://www.nacion.com/2010-0826/EIPais/UltimaHora/EIPais2499923.aspx

Mata, A. (27 de agosto 2010b). Puertos volverán a cerrar once días al año. La Nación. Recuperado de http://www.nacion.com/2010-0827/EIPais/NotaPrincipal/EIPais2499879.aspx

Mata, A. (30 de agosto 2010c). Sindicalistas de Japdeva y Gobierno inician conversaciones. La Nación. Recuperado dehttp://www.nacion.com/2010-0830/EIPais/UltimaHora/EIPais2504604.aspx 
Mata, A. (30 de agosto 2010d). Gobierno y muelleros evitan temas conflictivos en primera reunión. La Nación. Recuperado de http://www.nacion.com/2010-0831/EIPais/NotasSecundarias/EIPais2504445.aspx

Mata, A y Loaiza, V. (28 de octubre 2010) Puertos de Limón volverán a cerrar once días al año. La Nación. Recuperado de http://www.nacion.com/2010-0827/EIPais/NotaPrincipal/EIPais2499879.aspx

McQuail, D. (1996) Introducción a la teoría de la comunicación de masas (2a Ed.). México: Paidós Comunicación. Recuperado de http://www.planetadelibros.com/introduccion-a-la-teoria-de-la-comunicacion-demasas-libro-19121.html

Morera H, D. (2 de diciembre 2009) La intervención del Ministerio de Trabajo en el sindicato de JAPDEVA. Semanario Universidad. Recuperado de http://semanariouniversidad.ucr.cr/opinion/la-intervencin-del-ministerio-de-trabajoen-el-sindicato-de-japdeva/

Morris, G. K. (9 de septiembre 2010b). Día clave para la apertura de muelles el 15 de septiembre. Diario Extra. Recuperado de http://www.diarioextra.com/2010/setiembre/08/nacionales08.php

Morris G. K. (28 de diciembre 2010c). Nueva plata no le alcanza a JAPDEVA para mejorar gestión. Diario Extra. Recuperado de http://www.diarioextra.com/2010/diciembre/28/nacionales12.php

Morris, G. K. (6 de enero 2011a). JAPDEVA hablará de incapacidades con la CCSS. Diario Extra. Recuperado de http://www.diarioextra.com/2011/enero/06/nacionales06.php

Morris, G. K. (17 de enero 2011b). Concesión de Muelles de Moín debe ser regulada. Diario Extra. Recuperado de http://www.diarioextra.com/2011/enero/17/nacionales06.php

Morris, G. K. (19 de febrero 2011c). Los Muelles de Limón deben quedar en manos del Estado. Diario Extra. Recuperado de http://www.diarioextra.com/2011/enero/19/nacionales03.php

Murillo, A. (22 de enero 2011). Grupo perdedor en Japdeva culpa al Gobierno. La Nación. Recuperado de http://www.nacion.com/2011-0123/EIPais/NotasSecundarias/EIPais2660109.aspx

REDACCION (26 de agosto 2010). Trabajadores divididos en Japdeva tras fallo de Sala IV. La Nación. Recuperado dehttp://www.nacion.com/2010-0826/ElPais/UltimaHora/EIPais2499800.aspx 
Rojas, R. (12 de junio 2009). Sindicalistas denuncian amenazas de Sintrajap. La Nación. Recuperado de http://www.nacion.com/nacional/servicios-publicos/Sindicalistasdenuncian-amenazas-Sintrajap $0 \quad 1055094520 . \mathrm{html}$

Ruíz, R, G. (28 de agosto 2010). Chinchilla con incertidumbre por convención de JAPDEVA. Diario Extra. Recuperado de http://www.diarioextra.com/2010/agosto/28/nacionales06.php

Shoemaker, P. J. y Reese, S. D. (1994) La mediatización del mensaje. Teorías de las influencias en el contenido de los medios de comunicación. México, Distrito Federal: Editorial Diana. Recuperado de https://goo.gl/sbSMM3

Villalobos R, M. (15 de junio 2009). Gobierno ofrece mordida a los empleados a cambio de JAPDEVA. Diario Extra. Recuperado de http://www.diarioextra.com/2009/junio/15/nacionales05.php

Vízcaino, I. (17 de febrero 2011) La palabra concesión murió para nosotros. La Nación. Recuperado de http://www.nacion.com/2011-0218/EIPais/NotasSecundarias/EIPais2687498.aspx 